\title{
Canonical stochastic web map
}

\author{
S. S. Abdullaev \\ Institut für Energieforschung-Plasmaphysik, Forschungszentrum Jülich GmbH, EURATOM Association, Trilateral Euregio Cluster, \\ D-52425 Jülich, Germany
}

(Received 16 May 2007; revised manuscript received 26 June 2007; published 28 August 2007)

\begin{abstract}
In many Hamiltonian systems subjected to a time (or space) -periodic perturbation with a broad spectrum an approach is widely used of replacing the system by periodically $\delta$-kicked dynamical systems, which allows us to reduce them to symplectic mappings. In this paper it is shown that this approach has a fundamental failure, and the corresponding mapping does not correctly describe the continuous original system. It is demonstrated on the example of the stochastic web map obtained by this approach to describe a periodically driven harmonic oscillator which exhibits non-Kolmogorov-Arnold-Moser chaos, particularly, the formation of stochastic webs. A correct canonical map corresponding to this system is obtained using a recently developed method based on the canonical transformation of variables [S. S. Abdullaev, J. Phys. A 35, 2811 (2002)]. Using a direct numerical integration of the system it is shown that the canonical map correctly describes the periodically driven harmonic oscillator with a finite number of spectrum modes.
\end{abstract}

DOI: 10.1103/PhysRevE.76.026216

PACS number(s): 05.45.Ac

A Poincaré map is a powerful tool to study dynamical systems, particularly Hamiltonian systems which describe many fundamental models of physical systems whenever dissipation is negligible. A method widely used to obtain these maps for Hamiltonian systems subjected to time (or space) -periodic perturbations with a broad spectrum is based on the so-called $\delta$-function method. In this method the timeperiodic perturbation is replaced by the periodic in time (or space) $\delta$ functions, i.e., $\delta$ kicks. This method was originally used in Refs. [1,2] to derive the standard (or ChirikovTaylor) map. Up to the present time it has been uncritically used in many physical problems, ranging from the problems plasma physics to dynamical astronomy (see references in [3]). As was shown recently [4,5] (see also the book [3] and arguments below) this method encounters some fundamental physical and mathematical difficulties. In particular cases, it yields maps which are noncanonical and whose variables do not coincide with the variables of the original system.

To demonstrate these difficulties we consider a problem of the periodically driven harmonic oscillator. This is an important and interesting problem exhibiting nonKolmogorov-Arnold-Moser chaos and, in particular, the formation of stochastic webs with a regular quasicrystalline structures. Such a chaotic dynamics occurs in many physical problems like the stochastic particle acceleration in space and laboratory plasmas [6-11], passive particle transport in laminar flows [12], turbulence, quasicrystals, etc. (see Refs. [13-15], and references therein). Moreover, in recent experiments $[15,16]$ the effect of stochastic webs on chaotic electron transport in superlattices has been found.

A stochastic web structure has been first discovered by Zaslavsky et al. [17] while studying the chaotic dynamics of a particle in a magnetic field affected by a wave packet. This problem has been studied by the stochastic web map (SWM) obtained by replacing a periodically driven harmonic oscillator to a $\delta$-kicked system, and integrating the corresponding equations of motion with "delta-function kicks." The generalization of the SWM to the relativistic case has been given in $[18,19]$.

The main purpose of this work is to show the fundamental failures of the $\delta$-function method on the example of the periodically driven harmonic oscillator and to obtain the correct map for this system. For this we employ a recently developed method [3-5] to construct mappings for Hamiltonian systems based on the canonical transformation of variables.

Consider the dynamics of a charged particle of mass $m$ in the $(x, y)$ plane in a constant magnetic field $B_{0}$ along the $z$ axis and a wave field with a broad frequency spectrum propagating along the $y$ axis. In dimensionless variables, a dimensionless coordinate $\xi=k\left(y+P_{x} / m \omega_{0}\right)$, a momentum $\eta$ $=\left(k / m \omega_{0}\right) p_{y}$, a time $\tau=t(\Omega / 2 \pi)$, where $\omega_{0}=e B_{0} / m c$ is a gyrofrequency, the system is reduced to the dynamics of the harmonic oscillator driven by a wave field,

$$
\dot{\xi}=\eta, \quad \dot{\eta}=-\omega_{0}^{2} \xi+K \sum_{s=-M}^{M} \sin (\xi-2 \pi s \tau),
$$

where $K=2 \pi e \phi_{0} k^{2} /\left(m \omega_{0} \Omega\right)$ is a dimensionless perturbation parameter, expressed through the amplitude $\phi_{0}$, the wave number $k$, and the fundamental frequency $\Omega$ of the wave field, respectively, and $M$ is a number of maximal harmonics. The Hamiltonian corresponding to the system (1) is

$$
H=\frac{\alpha}{2}\left(\eta^{2}+\xi^{2}\right)+K \sum_{s=-M}^{M} \cos (\xi-2 \pi s \tau),
$$

with the canonical variables $(\xi, \eta)$. Here $\alpha=2 \pi \omega_{0} / \Omega$ is a winding number.

The evolution of the Hamiltonian system (1) and (2) in the case of infinite number of modes $M$ has been studied by Zaslavsky et al. [17] (see also [14]). Using the Poisson summation rule,

$$
\sum_{s=-\infty}^{\infty} \cos (2 \pi s \tau)=\sum_{k=-\infty}^{\infty} \delta(\tau-k)
$$

the corresponding equations of motion were integrated over $\delta$ functions, and the dynamics of the system was reduced to the stochastic web map (SWM), 


$$
\begin{gathered}
\eta_{k+1}=\left(\eta_{k}+K \sin \xi_{k}\right) \cos \alpha+\xi_{k} \sin \alpha, \\
\xi_{k+1}=-\left(\eta_{k}+K \sin \xi_{k}\right) \sin \alpha+\xi_{k} \cos \alpha,
\end{gathered}
$$

where $\eta_{k}=\eta(k-0), \xi_{k}=\xi(k-0)$ are the value of $\eta$ and $\xi$ just before the " $\delta$ kick" at the time instant $\tau=k$.

The generalization of the map (3) to the relativistic case has been obtained by Longcope and Sudan [18]: it is given by the map similar to Eq. (3) with the parameter $\alpha$ is replaced by $\alpha / \gamma$, where $\gamma^{2}=1+\beta^{2}\left[\left(\eta_{k}+K \sin \xi_{k}\right)^{2}+\xi_{k}^{2}\right], \quad \beta$ $=\omega_{0} / k c$.

As was mentioned above the derivation of mapping for such systems based on the $\delta$ function approach is incorrect from the mathematical point of view as well as from the physical consideration (see Ref. [3] and references therein). In real physical situations a broad spectrum of wave frequencies means that mode number $M$ in the sum (2) is large but finite. The formal replacement of the finite sum of trigonometric functions with the infinite sum of delta functions leads to the complete loss of information on the system's original variables and the new system is not defined at the discrete time instants, $\tau=k$. Moreover, the integration of the equations of motion over the $\delta$ functions is not always a welldefined procedure.

The above procedure can be rigorously done only by the canonical change of variables in the Hamiltonian system. In the limit $M \rightarrow \infty$ the generating function implementing the change of variables vanishes at all times except the periodic time instants $\tau=k$ where the system variables are measured. Therefore the variables of the mapping (3) will be different from the ones in the original system. The difference between these, old and new variables, can reach the magnitude of the perturbation parameter $K$, which can be of order of unity.

Below we use the method of canonical transformations of variables to construct mappings recently developed in Refs. $[3-5,20]$ to derive a mapping for the Hamiltonian system (2). The idea of constructing mappings for the broad spectrum perturbation consists of two steps: first one obtains the mapping for the finite number mode $M$, and then one takes the limit $M \rightarrow \infty$ to simplify the mapping. According to a locality property (see Ref. [21]) the obtained map should correctly describe the system with a finite number of modes $M$ in the physical domain of variables $(\xi, \eta)$ where the resonances of type $m \omega=s \Omega$ with $|s|<M$ occurs ( $\omega$ is a frequency of motion).

We introduce the new canonical action-angle variables $(\vartheta, I)$, related to the old variables $(\xi, \eta)$ as $\xi$ $=(2 I)^{1 / 2} \sin \vartheta, \eta=(2 I)^{1 / 2} \cos \vartheta$, and rewrite the Hamiltonian (2) in the form

$$
\begin{gathered}
H=\alpha I+\epsilon H_{1}(I, \vartheta, \tau), \\
\epsilon H_{1}(I, \vartheta, \tau)=K \sum_{s=-M}^{M} \cos (\sqrt{2 I} \sin \vartheta-2 \pi s \tau) \\
=K \sum_{s=-M}^{M} \sum_{n=-\infty}^{\infty} J_{n}(\sqrt{2 I}) \cos (n \vartheta-2 \pi s \tau),
\end{gathered}
$$

where $H_{1}(I, \vartheta, \tau)$ describes the effect of a wave packet on the harmonic oscillator. The latter is not required to be small.

Let $\left(\vartheta_{k}, I_{k}\right)=\left(\vartheta\left(\tau_{k}\right), I\left(\tau_{k}\right)\right)$ be values the variables $(\vartheta(\tau), I(\tau))$ taken at the discrete time instances $\tau_{k}=k,(k$ $=0, \pm 1, \pm 2, \ldots)$. We construct a mapping

$$
\left(\vartheta_{k+1}, I_{k+1}\right)=\hat{T}\left(\vartheta_{k}, I_{k}\right),
$$

relating variables $\left(\vartheta_{k}, I_{k}\right)$ at the time instant $\tau_{k}$ with ones $\left(\vartheta_{k+1}, I_{k+1}\right)$ at the time instance $\tau_{k+1}$.

The mapping (5) can be constructed in the following symplectic form [3-5]:

$$
\begin{gathered}
\Theta_{k}=\vartheta_{k}+\epsilon \frac{\partial S_{k}}{\partial J_{k}}, \quad J_{k}=I_{k}-\epsilon \frac{\partial S_{k}}{\partial \vartheta_{k}}, \\
\bar{\Theta}_{k}=\Theta_{k}+w\left(J_{k}\right), \\
\vartheta_{k+1}=\bar{\Theta}_{k}-\epsilon \frac{\partial S_{k+1}}{\partial J_{k}}, \quad I_{k+1}=J_{k}+\epsilon \frac{\partial S_{k+1}}{\partial \vartheta_{k+1}},
\end{gathered}
$$

where $w\left(J_{k}\right)$ is perturbed frequency, $S_{k}=S\left(\vartheta_{k}, J_{k}, \tau_{k}, \tau_{0}\right)$, $S_{k+1}=S\left(\vartheta_{k+1}, J_{k}, \tau_{k+1}, \tau_{0}\right)$ are values of the generating function $S\left(\vartheta, J, \tau, \tau_{0}\right)$ at the time instants $\tau=\tau_{k}$ and $\tau=\tau_{k+1}$, respectively. The latter obeying the Hamilton-Jacobi equation is found as a series of powers of $\epsilon: S=S_{1}+\epsilon S_{2}+\cdots$ using the perturbation theory in a finite time interval $\tau_{k}<\tau<\tau_{k+1}$. In the first order of the perturbation theory one obtains

$$
\begin{aligned}
S_{1}\left(\vartheta, J, \tau, \tau_{0}\right)= & -\left(\tau-\tau_{0}\right) K \sum_{s=-M} \sum_{n=-\infty}^{\infty} J_{n}(\sqrt{2 J}) \\
& \times\left[a\left(x_{n s}\right) \sin \Psi_{n s}+b\left(x_{n s}\right) \cos \Psi_{n s}\right],
\end{aligned}
$$

where $\Psi_{n s}=n \vartheta-2 \pi s \tau, \quad a(x)=(1-\cos x) / x, \quad b(x)=\sin x / x$, $x_{n s}=n \omega(J)-2 \pi s, \tau_{k}<\tau_{0}<\tau_{k+1}$.

As was shown in Refs. [3,20] at the limit $M \rightarrow \infty$ the higher order corrections $S_{i},(i \geqslant 2)$, to the generating function vanish and the mapping (6) becomes exact with the generating function

$$
\epsilon S_{k}=-\epsilon S_{k+1}=\frac{K}{2} \cos (\sqrt{2 J} \sin \vartheta) .
$$

Also one has $w(J)=\alpha$. Using Eq. (8), we obtain

$$
\begin{gathered}
\Theta_{k}=\vartheta_{k}-\frac{K}{2 \sqrt{2 J_{k}}} \sin \left(\sqrt{2 J_{k}} \sin \vartheta_{k}\right) \sin \vartheta_{k}, \\
J_{k}=I_{k}+\frac{K}{2} \sqrt{2 J_{k}} \sin \left(\sqrt{2 J_{k}} \sin \vartheta_{k}\right) \cos \vartheta_{k}, \\
\bar{\Theta}_{k}=\Theta_{k}+\alpha, \\
\vartheta_{k+1}=\bar{\Theta}_{k}-\frac{K}{2 \sqrt{2 J_{k}}} \sin \left(\sqrt{2 J_{k}} \sin \vartheta_{k+1}\right) \sin \vartheta_{k+1}, \\
I_{k+1}=J_{k}+\frac{K}{2} \sqrt{2 J_{k}} \sin \left(\sqrt{2 J_{k}} \sin \vartheta_{k+1}\right) \cos \vartheta_{k+1} .
\end{gathered}
$$


Furthermore, we call the mapping (9) a canonical stochastic web map (CSWM). This mapping is valid for the arbitrary value of the perturbation parameter $K$. In contrast to the SWM (3) it is implicit with respect to the variables $J_{k}$ and $\vartheta_{k+1}$. It can be also considered as the integration method of Hamiltonian system (2) and (4) with infinite number of modes $M$.

It is known that for the values $\alpha=2 \pi / q$ with integer numbers $q$ the system (2) generates the crystalline structures in the phase space $(\xi, \eta)$ if $q=2,3,4,6$, or quasicrystalline structures if $q=5,7,8, \ldots$ [17]. Such structures were called stochastic webs.

We have compared the CSWM with the direct numerical integration of the original Hamiltonian system (2) with a finite number of modes $M$ using the standard symplectic small-step integration scheme [22]. As was noted above according to a locality property in a finite area of phase space $(\xi, \eta)$ near the its origin $(\xi=0, \eta=0)$ the behavior of the system (2) with a finite number of modes $M$ should be close to the one with $M \rightarrow \infty$. Figure 1 shows the phase space of the system (2) for $q=6$ obtained by the CSWM (a), SWM (b), and the comparison of them with the direct numerical integration with a finite mode number $M=5$ (c). The integration step is taken equal to $\Delta t=10^{-2}$ and the perturbation parameter $K=1$. At the first glance one notices that the CSWM creates the stochastic web along the separatrices of the averaged Hamiltonian (see [13]),

$$
\bar{H}=(K / q) \sum_{j=1}^{q} \cos \left(\mathbf{e}_{j} \cdot \mathbf{r}\right),
$$

corresponding to the system (4) at $\alpha=2 \pi / q$ and $\beta=0$. Here $\mathbf{e}_{j}=(\cos (2 \pi j / q), \sin (2 \pi j / q)), \mathbf{r}=(\xi, \eta)$. The SWM forms the stochastic layer which strongly deformed from the unperturbed separatrices. This deformation is not due to the effect of perturbation as stated in Ref. [23], but related to the fact that the variables of the SWM are nonidentical with ones of the original system (2). It means the estimations of the width of the stochastic layer made in several works [23-25] using the separatrix-splitting of the averaged Hamiltonian cannot be, in general, compared with ones of the SWM. The agreement between such estimations and the SWM obtained in these works for the case $q=4$, as we will see below, occurs by coincidence since the CSWM and the SWM create the stochastic layers of comparable widths. However, in the cases $q=3,6$ no rigorous estimations have been obtained.

As seen from Fig. 1(c) the CSWM (9) [blue (dark gray) lines] is much closer to the original Hamiltonian system (2) [orange (gray) lines] than the SWM (3) [green (light gray) dots]. The latter creates the stochastic layer which has a width of several orders larger than one for the original system. The stochastic layer of the original system for the perturbation parameter $K=1$ with a finite mode number $M$ is negligibly small, which agrees with the prediction of the CSWM.

The cases $q=4$ and $q=3$ are shown in Figs. 2 and 3, respectively. In the case of $q=4$ (Fig. 2) the widths of the stochastic layer created by the CSWM (a) and the SWM are comparable. However, for $q=3$ there is a strong difference
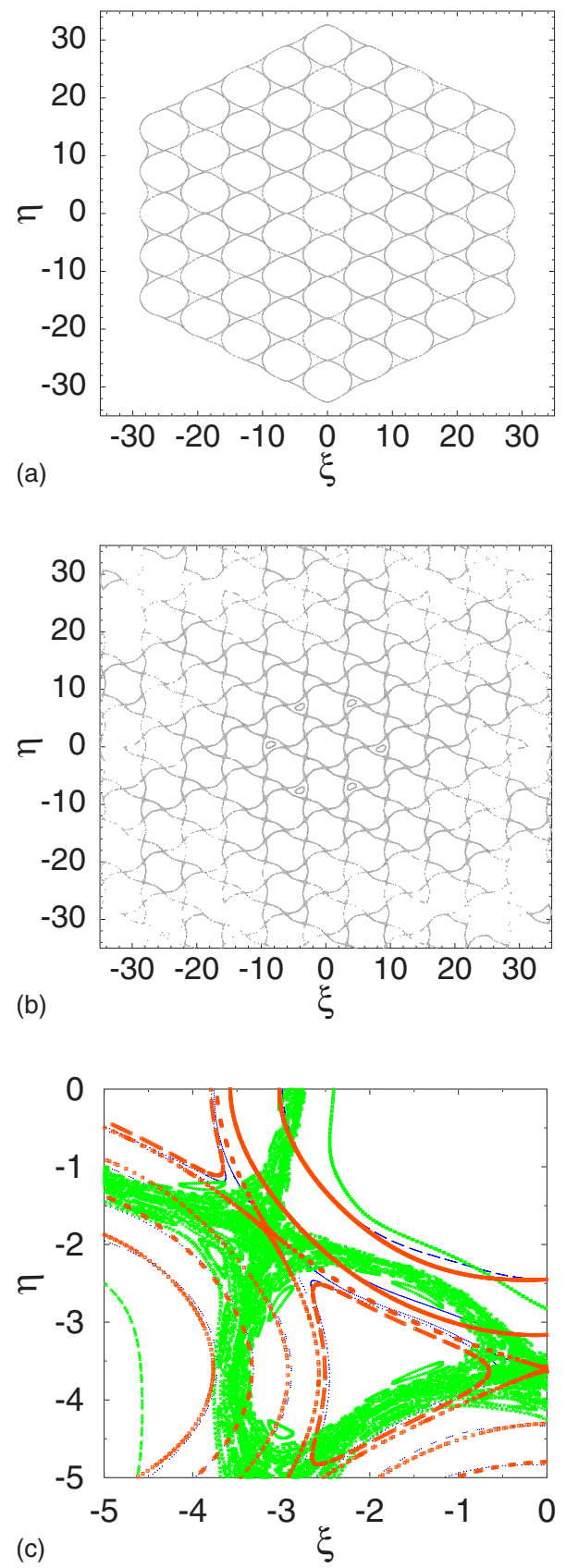

FIG. 1. (Color online). Phase spaces of the CSWM (9) (a) and the SWM (3) (b) obtained with the same initial conditions; (c) comparison of phase spaces obtained by the CSWM (9) [blue (dark gray) lines], the SWM [green (light gray) dots] and the small-step numerical integration of the system (2) with a finite $M=5$ [orange (gray) lines]. The time step of integration $\Delta t=10^{-2}$. Other parameters are $q=6, K=1$.

between the stochastic layers of the SWM and the original system [Fig. 3(a)] which is close to the one of the CSWM [Fig. 3(b)]. The SWM creates a negligibly small stochastic layer, opposite to the case $q=6$.

The CSWM also predicts a new feature in the chaotic transport regime along the stochastic web which was not observed in the SWM dynamics. Particularly, it was found that for the case $q=6$ the second moment of displacement in 

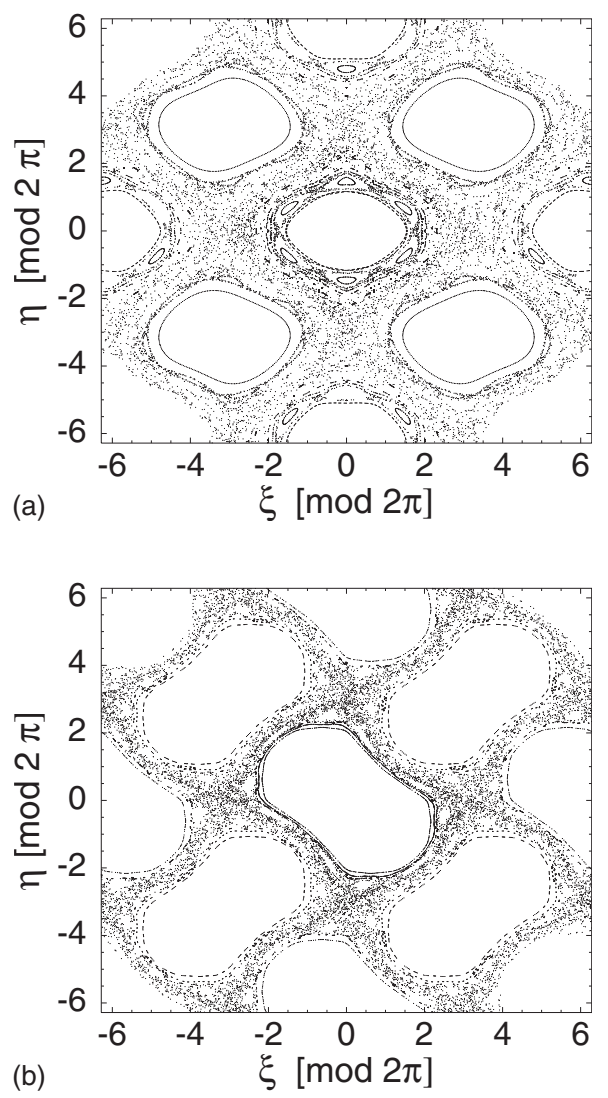

FIG. 2. Phase space of the CSWM (a) and the SWM (b). Parameters are $\alpha=2 \pi / 4, K=1.5$.

the phase space, $\sigma^{2}(k)=\left\langle\xi_{k}^{2}+\eta_{k}^{2}\right\rangle$, has the following asymptotics at large time steps $k, \sigma^{2}(k)=2 D k^{\gamma}$ with the exponent $\gamma=2.3-1.4$ for the values of $K$ in the interval $1.4<K<1.6$. Such acceleration regimes has not been observed in the SWM chaotic dynamics. For the perturbation parameter $K$ in the interval $1.4<K<1.6$ the SWM mostly exhibits the subdiffusive transport with the exponent $\gamma \approx 0.8-0.9$.

The CSWM (9) can be easily generalized to the relativistic case: one should simply replace $\alpha$ to $\alpha / \sqrt{1+2 \beta^{2} J_{k}}$. In this case the stochastic web is formed only in a finite area of the phase space [18].

In conclusion, in the examples of periodically driven harmonic oscillators it is shown that the derivation of Poincaré maps by the replacement of Hamiltonian systems subjected to time-periodic perturbation with a broad spectrum with periodic $\delta$-kicked systems is not correct from the physical and mathematical points of view. Such replacement results, first, in changing of variables of the original continuous system, and, second, encounters the ill-defined mathematical proce-
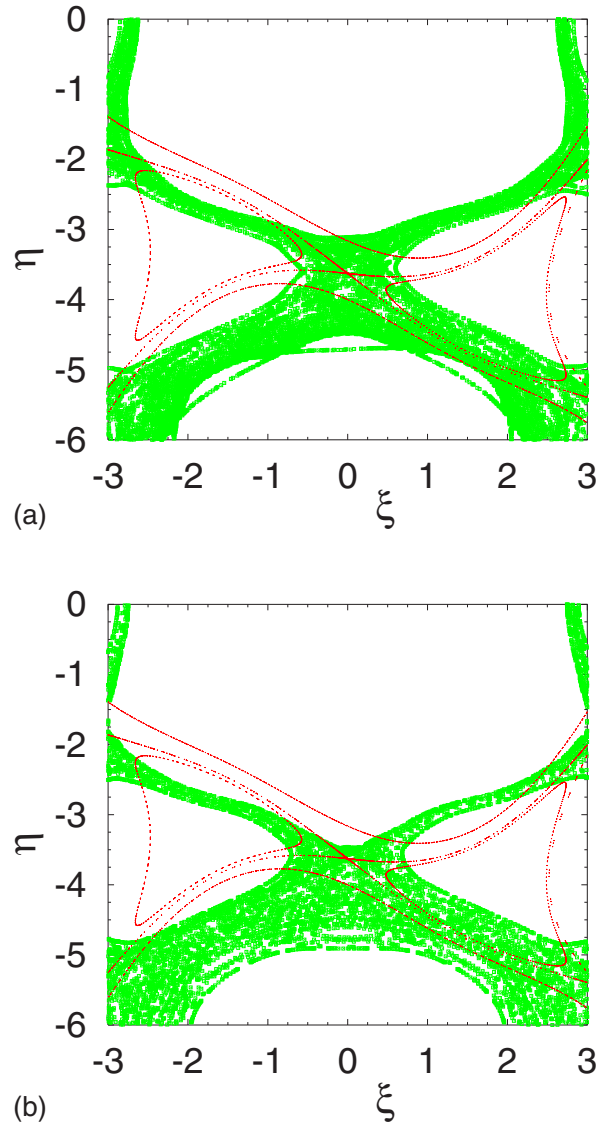

FIG. 3. (Color online). Comparison the SWM (3) [red (dark gray) lines] with the small step symplectic integration of the Hamiltonian system (2) [green (light gray) dots in (a)] and the CSWM (9) [green (light gray) dots in (b)]. The time step of integration $\Delta t$ $=10^{-2}$ and $M=5$. Parameters are $\alpha=2 \pi / 3, K=1$.

dure of integration of $\delta$-kicked systems over $\delta$ functions. The correct map whose variables coincide with ones of the original system can be obtained by the method based on the canonical transformation of variables in dynamical equations. We have derived the canonical map, CSWM, describing the dynamics of the harmonic oscillator driven by the wave field with a broad frequency spectrum. It is shown that the CSWM correctly describes the properties of the stochastic web of the original system with a finite mode number, unlike the SWM derived using the $\delta$-kicked systems.

The CSWM can be easily generalized to the case with obliquely propagating waves considered in [19]. The CSWM and its generalizations can be used as a powerful tool to study the regular and chaotic dynamics of broad range physical systems exhibiting non-KAM chaos which were mentioned above.
[1] G. M. Zaslavsky and B. V. Chirikov, Sov. Phys. Usp. 14, 549 (1971).

[2] B. V. Chirikov, Phys. Rep. 52, 265 (1979).

[3] S. S. Abdullaev, Construction of Mappings for Hamiltonian
Systems and Their Applications, Lecture Notes in Physics Vol. 691 (Springer-Verlag, Berlin, Heidelberg, 2006).

[4] S. S. Abdullaev, J. Phys. A 32, 2745 (1999).

[5] S. S. Abdullaev, J. Phys. A 35, 2811 (2002). 
[6] G. R. Smith and A. N. Kaufman, Phys. Rev. Lett. 34, 1613 (1975).

[7] C. F. F. Karney and A. Bers, Phys. Rev. Lett. 39, 550 (1977).

[8] C. F. F. Karney, Phys. Fluids 21, 1584 (1978).

[9] C. F. F. Karney, Phys. Fluids 22, 2188 (1979).

[10] C. P.-K., L. Schmitz, and R. W. Conn, Phys. Plasmas 3, 1545 (1996).

[11] A. K. Ram, A. Bers, and D. Benisti, J. Geophys. Res. 103, 9431 (1998).

[12] A. A. Chernikov, B. A. Petrovichev, A. V. Rogal'sky, R. Z. Sagdeev, and G. M. Zaslavsky, Phys. Lett. A 144, 127 (1989).

[13] G. M. Zaslavsky, Chaos 1, 1 (1991).

[14] G. M. Zaslavsky, R. V. Sagdeev, D. A. Usikov, and A. A. Chernikov, Weak Chaos and Quasi-regular Patterns (Cambridge University Press, Cambridge, England, 1991).

[15] T. M. Fromhold et al., Nature (London) 428, 726 (2004).

[16] T. M. Fromhold, A. A. Krokhin, C. R. Tench, S. Bujkiewicz, P.
B. Wilkinson, F. W. Shea, and L. Eaves, Phys. Rev. Lett. 87, 046803 (2001).

[17] G. M. Zaslavsky, M. Y. Zakharov, R. Z. Sagdeev, D. A. Usikov, and A. A. Chernikov, Sov. Phys. JETP 64, 294 (1986).

[18] D. W. Longcope and R. N. Sudan, Phys. Rev. Lett. 59, 1500 (1987).

[19] H. Karimabadi and V. Angelopoulos, Phys. Rev. Lett. 62, 2342 (1989).

[20] S. S. Abdullaev, Nucl. Fusion 44, S12 (2004).

[21] Y. Elskens and D. F. Escande, Microscopic Dynamics of Plasmas and Chaos (Institute of Physics, Bristol, 2003).

[22] R. McLachlan and P. Atela, Nonlinearity 5, 541 (1992).

[23] V. V. Afanasiev, A. A. Chernikov, R. Z. Sagdeev, and G. M. Zaslavsky, Phys. Lett. A 144, 229 (1990).

[24] A. J. Lichtenberg and B. Wood, Phys. Rev. A 39, 2153 (1989).

[25] S. Pekarsky and V. Rom-Kedar, Phys. Lett. A 225, 274 (1997). 